\title{
Positron-electron autocorrelation function study of E-center in silicon
}

\author{
K. F. Ho, H. M. Ching, C. D. Beling, and S. Fung ${ }^{\text {a) }}$ \\ Department of Physics, The University of Hong Kong, Pokfulam Road, Hong Kong, \\ People's Republic of China \\ K. P. Ng \\ Department of Mathematics, The University of Hong Kong, Pokfulam Road, Hong Kong, \\ People's Republic of China \\ M. Biasini and G. Ferro \\ ENEA, Via don Fiammelli 2, IT-401128 Bologna, Italy \\ M. Gong \\ Department of Physics, Sichuan University, Chengdu, Sichuan 610064, People's Republic of China
}

(Received 3 March 2003; accepted 4 August 2003)

Two-dimensional angular correlation of annihilation radiation (2D-ACAR) spectra have been taken for $10^{19} \mathrm{~cm}^{-3}$ phosphorus-doped $\mathrm{Si}$ in the as-grown state after having been subjected to $1.8 \mathrm{MeV}$ electron fluences of $1 \times 10^{18}$ and $2 \times 10^{18} \mathrm{~cm}^{-2}$. Positron annihilation lifetime spectroscopy confirms, in accordance with previous works, that positrons are saturation trapping into $\left(\mathrm{V}_{\mathrm{Si}}: \mathrm{P}\right)$ pair defect (E-center) monovacancy sites in the electron irradiated samples. In the as-grown case, the positron-electron autocorrelation functions along the [111] and [1-10] directions, obtained through Fourier transformation of the 2D-ACAR data, reveal zero-crossings that deviate only slightly from the lattice points, in a manner consistent with positron-electron correlation effects. Conversely, in the spectra of the irradiated samples, the zero-crossing points are observed to move outward further by between 0.15 and $0.50 \AA$. This displacement is associated with positron annihilation with electrons in localized orbitals at the defect site. An attempt is made to extract just the component of the defect's positron-electron autocorrelation function that relates to the localized defect orbitals. In doing this features are found that correspond to the expected atomic positions at the vacancy defect site suggesting that this real-space function may provide a convenient means for obtaining a mapping of localized orbitals. The observed approximate separability of positron and electron wave-function autocorrelates leads to an estimate of $0.22 \mathrm{eV}$ for the positron binding energy to the E-center. () 2003 American Institute of Physics. [DOI: 10.1063/1.1613368]

\section{INTRODUCTION}

Five years ago McMullen and Bishop pointed out that the two-dimensional angular correlation of annihilation radiation (2D-ACAR) spectrum of a positron in a defect could be considered as a kind of "diffraction pattern" arising from annihilations with localized defect orbitals. ${ }^{1}$ In analogy with the form factor in atomic and nuclear elastic scattering the speculation naturally arises as to whether or not the "diffraction pattern" can in some sense be inverted to give some kind of spatial distribution of the electron-positron wave function at the defect site with the potentiality perhaps of some new atomic scale defect microscopy. It was shown that this idea was closely linked to the concept of the 2D-ACAR spectra being the Fourier transform of planar cut through the positron-electron autocorrelation function $B^{2 \gamma}(\mathbf{r}),{ }^{2}$ a fact that had been known since the early $1980 \mathrm{~s}^{3,4}$ due to the close parallels with Compton profile momentum spectroscopy., Close inspection of the inversion problem suggests that the $B^{2 \gamma}(\mathbf{r})$ function of a defect may indeed be the most reduced form that quantum mechanics permits for microscopically

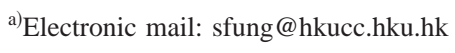

"imaging" the localized electron orbitals at the defect site. ${ }^{2}$ In this article we explore this suggestion further with specific reference to the E-center in silicon.

The photon pair momentum density $\rho^{2 \gamma}(\mathbf{p})$ in a positron annihilation experiment under the independent particle model (IPM) approximation is given by ${ }^{7}$

$$
\rho^{2 \gamma}(\mathbf{p})=\sum_{j}\left|\mathrm{FT}_{-3}\left[\psi_{+, j}(\mathbf{r})\right]\right|^{2},
$$

where $\mathrm{FT}_{-3}$ is the operation of three-dimensional Fourier transformation and $\psi_{+, j}(\mathbf{r})=\psi_{+}(\mathbf{r}) \psi_{j}(\mathbf{r})$ is the positronelectron wave function, the summation being taken over all $j$ electron states of the band structure. Another equally valid representation containing exactly the same information as $\rho^{2 \gamma}(\mathbf{p})$ is the positron-electron autocorrelation function, $B^{2 \gamma}(\mathbf{r})$, which is given by the Fourier transform of $\rho^{2 \gamma}(\mathbf{p})$. Using the convolution theorem one can easily see that

$$
\begin{aligned}
B^{2 \gamma}(\mathbf{r}) & =\mathrm{FT}_{-3}\left\{\rho^{2 \gamma}(\mathbf{p})\right\}=\mathrm{FT}_{-3}\left\{\left.\sum_{j}\left|\mathrm{FT}_{-3}\left[\psi_{+, j}(\mathbf{r})\right]\right|\right|^{2}\right\} \\
& =\mathrm{FT}_{-3}\left\{\sum_{j} \mathrm{FT}_{-3} \int \psi_{+, j}^{*}(\mathbf{r}+\mathbf{s}) \psi_{+, j}(\mathbf{r}) d \mathbf{s}\right\} \\
& =\sum_{j} \int \psi_{+, j}^{*}(\mathbf{r}+\mathbf{s}) \psi_{+, j}(\mathbf{s}) d \mathbf{s},
\end{aligned}
$$


from which it may be further seen that $B^{2 \gamma}(\mathbf{r})$ is really the sum over all positron-electron wave-function autocorrelates of respective electron states. This representation at first sight may seem to be less informative than that of $\rho^{2 \gamma}(\mathbf{p})$. However, when applied to positron annihilation with the localized defect electron orbitals that arise from dangling bonds $B^{2 \gamma}(\mathbf{r})$ is possibly the best function that diffraction pattern inversion permits for spatially mapping the location of such orbitals. This may be seen by observing the function

$$
\begin{aligned}
B_{\mathrm{loc}}^{2 \gamma}(\mathbf{r}) & =\sum_{j=1}^{n_{\mathrm{loc}}} \int \psi_{+\mathrm{loc}, j}^{*}(\mathbf{r}+\mathbf{s}) \psi_{+\mathrm{loc}, j}(\mathbf{s}) d \mathbf{s} \\
& =\mathrm{FT}_{-3}\left\{\sum_{j=1}^{n_{\mathrm{loc}}}\left|\mathrm{FT}_{-3}\left[\psi_{+, j}(\mathbf{r})\right]\right| 2\right\},
\end{aligned}
$$

in which the summation is just over the $n_{\text {loc }}$ localized electron orbitals associated with the defect, the $\psi_{+ \text {loc }, j \text { loc }}(\mathbf{r})$ $=\psi_{+\mathrm{loc}}(\mathbf{r}) \psi_{j \mathrm{loc}}(\mathbf{r})$ being the localized positron-electron wave function, $\psi_{+ \text {loc }}(\mathbf{r})$ and $\psi_{j \text { loc }}(\mathbf{r})$ being, respectively, the wave functions of the trapped positron and the $j$ th dangling bond electron. The last term of Eq. (3) shows clearly the diffraction integrals of type suggested by McMullin and Bishop $^{1}$ in which the aperture functions are the localized positron-electron wave functions $\psi_{+, j}(\mathbf{r})$. Unfortunately, as we shall see from the present work, $B_{\mathrm{loc}}^{2 \gamma}(\mathbf{r})$ is not a direct observable, but nonetheless the nature of Eq. (3) stands as a strong motivation for trying to extract this function or something closely related to it.

When applied to positron annihilation between a delocalized positron and Bloch state electrons in a regular semiconductor crystal $B^{2 \gamma}(\mathbf{r})$ reveals a remarkable property, namely that the function undergoes zero crossings when $\mathbf{r}$ is very close to a lattice vector $\mathbf{R}$. To understand why this is so one first writes Eq. (2) in the form ${ }^{3}$

$$
B^{2 \gamma}(\mathbf{r})=\sum_{j=1}^{n_{\text {val }}} e^{-i \mathbf{k} \cdot \mathbf{r}} \int u_{+, j}^{*}(\mathbf{r}+\mathbf{s}) u_{+, j}(\mathbf{s}) d \mathbf{s},
$$

from which it will be seen that under the condition that $\mathbf{r}$ $=\mathbf{R}_{\mathbf{L}}$ the integral in Eq. (4) becomes maximal and equal to unity. However, rather than maximal $B^{2 \gamma}(\mathbf{r})$ values being found at Bravais lattice positions, zero values are observed instead. These arise through the multiplicative phase summation in Eq. (4), as shown by the phasor diagram of Fig. 1. When $\mathbf{r}=0$ there is no phase difference between terms and a maxima is seen in $B^{2 \gamma}(\mathbf{r})$. When $\mathbf{r}=\mathbf{R}_{\mathbf{L}} / \mathbf{2}$ the real part of the summation is seen to be zero causing $B^{2 \gamma}(\mathbf{r})=0$ : the first zero crossing. The same situation arises for $\mathbf{r}=\mathbf{R}_{\mathbf{L}}$ and other multiples of $\mathbf{R}_{\mathbf{L}}$. Not only is the oscillatory nature of $B^{2 \gamma}(\mathbf{r})$ in the direction $\mathbf{R}_{\mathbf{L}}$ understood, but in addition Fig. 1 explains the general damped nature of the $B^{2 \gamma}(\mathbf{r})$ oscillations. This comes about as a result of there being fewer phase vectors to sum along the real axis after every lattice vector displacement $(2 \pi$ revolution in phase). This zero crossing theorem, while being exactly true for the case of the $B(\mathbf{r})$ function observed in Compton profile experiments, ${ }^{8,9}$ is not exactly true for the case of $B^{2 \gamma}(\mathbf{r})$. The reason is that

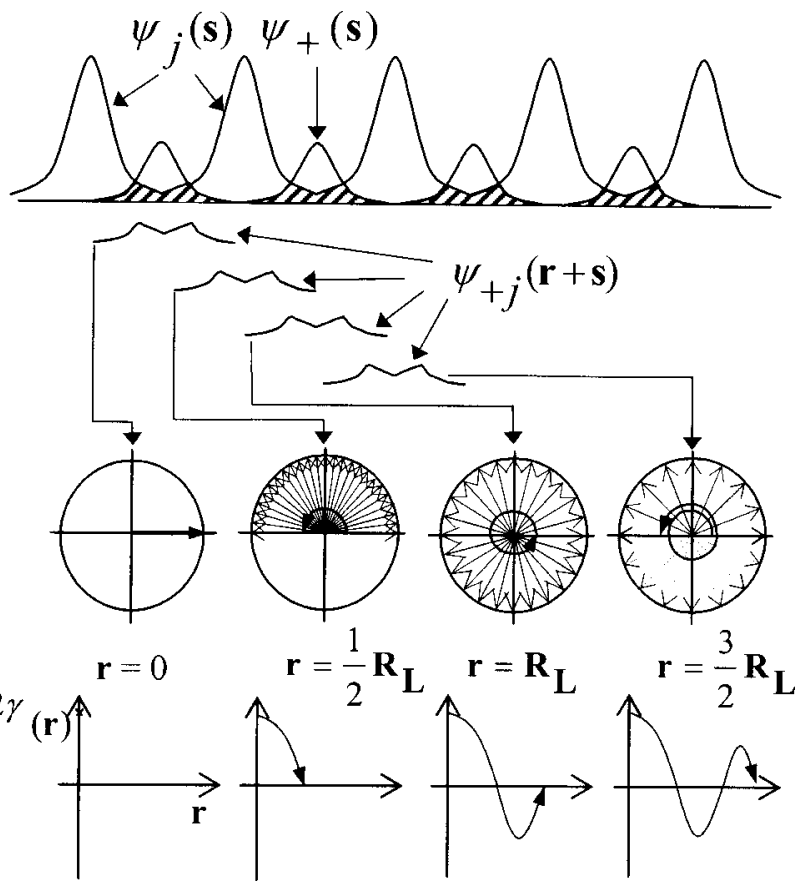

FIG. 1. Diagram showing how relative phasing of electron-positron Bloch waves causes zeros to occur in $B^{2 \gamma}(\mathbf{r})$ when $\mathbf{r}=\mathbf{R}_{\mathbf{L}}$. The upper part of the diagram shows the overlapping of the positron and electron Bloch waves (hatched region). The middle part of the diagram shows the relative phasing of the solid which assumes a simple lattice of 24 atoms (24 k-vectors per band) when $\mathbf{r}=0, \frac{1}{2} \mathbf{R}_{\mathbf{L}}, \mathbf{R}_{\mathbf{L}}$, and $\frac{3}{2} \mathbf{R}_{\mathbf{L}}$, respectively. The lower part shows the resulting $B^{2 \gamma}(\mathbf{r})$ function.

electron-positron correlation effects cause slight differences in the amplitudes of the summed phasors in Eq. (4).

Finally it is important to note that neither $\rho^{2 \gamma}(\mathbf{p})$ nor $B^{2 \gamma}(\mathbf{r})$ is directly accessible by experiment. Rather in a $2 \mathrm{D}-$ ACAR experiment the observable spectrum $N\left(p_{y}, p_{z}\right)$ is the 2D projection of $\rho^{2 \gamma}(\mathbf{p}):^{3}$

$$
\begin{aligned}
N\left(p_{y}, p_{z}\right) & =\text { const } \int_{-\infty}^{+\infty} \rho^{2 \gamma}(\mathbf{p}) d p_{x} \\
& =\text { const }_{\mathrm{FT}_{-2}}\left\{\left.B^{2 \gamma}(\mathbf{r})\right|_{x=0}\right\},
\end{aligned}
$$

from which follows that a planar section through $B^{2 \gamma}(\mathbf{r})$, namely $\left.B^{2 \gamma}(\mathbf{r})\right|_{x=0}=\left.B^{2 \gamma}(y, z)\right|_{x=0}$ defined for the crystallographic plane $(0, y, z)$, may be obtained directly from the Fourier transform of the 2D-ACAR data taken in the respective $p_{y}, p_{z}$ momentum directions. That is ${ }^{3}$

$$
\left.B^{2 \gamma}(y, z)\right|_{x=0} \propto \mathrm{FT}_{-2}\left\{N\left(p_{y}, p_{z}\right)\right\} .
$$

Following from the above discussion $\left.B^{2 \gamma}(y, z)\right|_{x=0}$ will display, for Bloch state positrons, zero values at Bravais lattice positions if they happen to lie on the chosen crystallographic plane $(0, y, z)$. On the other hand for trapped positrons at least some of the $\left.B^{2 \gamma}(y, z)\right|_{x=0}$ signal will arise from the presence (or perhaps more correctly the absence of) the autocorrelate signal of the localized defect electron orbitals as expressed in Eq. (3). The main emphasis in the present study is to try and ascertain the degree of involvement of localized electron orbitals in the forming of the $\left.B^{2 \gamma}(y, z)\right|_{x=0}$ signal and to attempt to extract that component of $\left.B^{2 \gamma}(y, z)\right|_{x=0}$ 

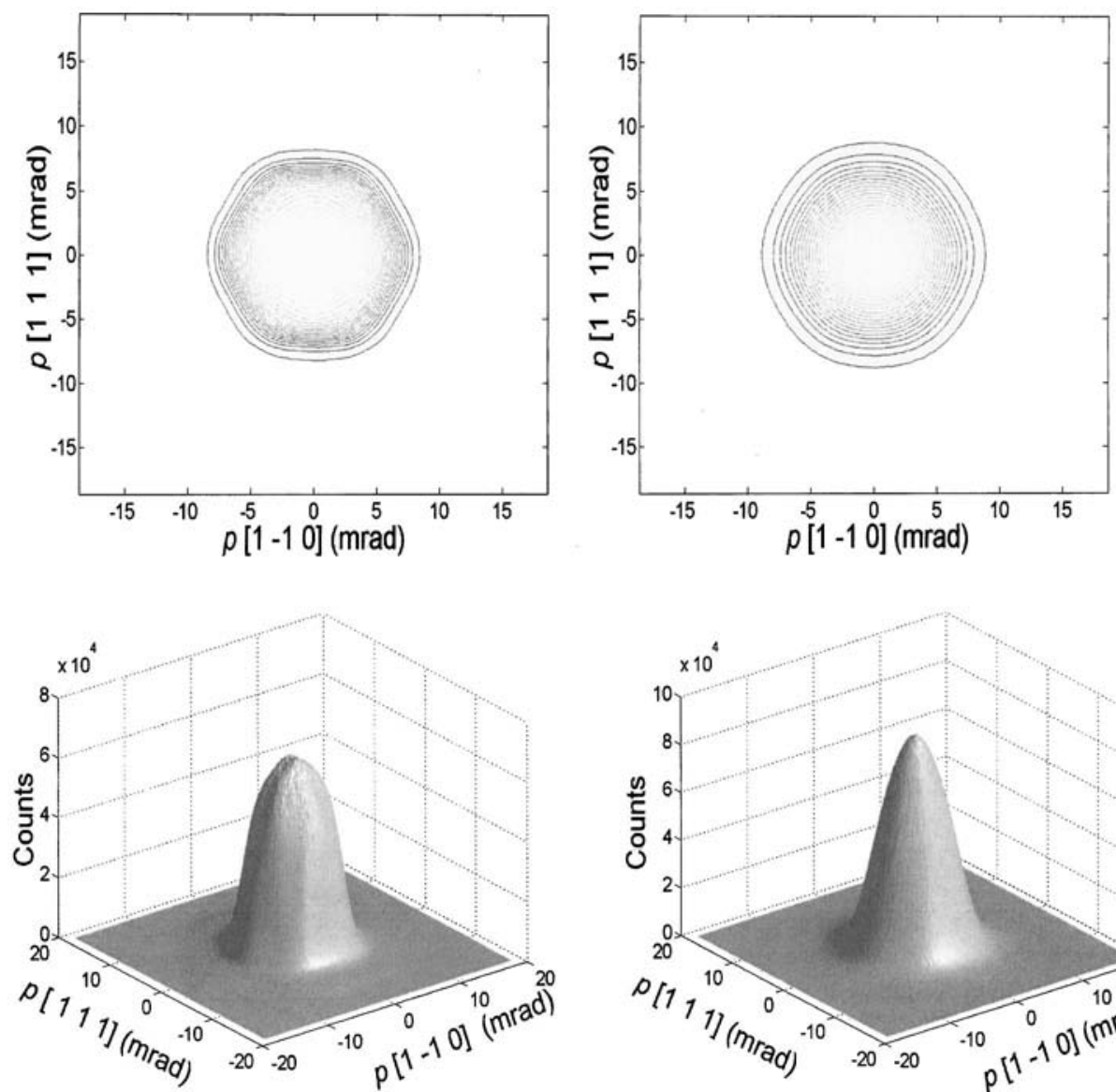

FIG. 2. 2D-ACAR data for (a) asgrown Si:P and (b) electron-irradiated Si:P in which saturation trapping into the E-center $\left(\mathrm{V}_{\mathrm{Si}}: \mathrm{P}\right.$ defect $)$ is occurring. which is related to the localized electron orbitals. In doing so a novel discovery is reported, namely that an estimate of the positron binding energy to the defect can be found by ratioing the positron-electron autocorrelates for bulk crystal and defect trapped positron states.

\section{EXPERIMENT}

The heavily $\mathrm{P}$ doped Si used in this study was purchased from Lattice Materials Corp., USA Ltd. and was (111) oriented. The wafers had a quoted resistivity in the range of $0.001-0.005 \Omega \mathrm{cm}$ [corresponding to a carrier concentration in the range $\left.(1-7) \times 10^{19} \mathrm{~cm}^{-3}\right]$. In accord with the study of Mäkinen et al. ${ }^{10} \mathrm{~V}_{\mathrm{Si}}$ :P type defects were introduced by subjecting the $\mathrm{Si}$ to $1.8 \mathrm{MeV}$ electron dosages of $1 \times 10^{18}$ and $2 \times 10^{18} e^{-} \mathrm{cm}^{-3}$ at room temperature using the LINAC facility at Sichuan University.

Positron annihilation lifetime spectroscopy (PALS) spectra were taken on the as-grown and defected samples using a fast-fast lifetime' spectrometer of 240 ps resolution. The 2DACAR spectrometer, at ENEA, Italy, used in the present work was of the standard design based on two Anger cameras, the details of which may be found in Biasini et al. ${ }^{11}$ The spectrometer had an average resolution of $\sim 0.75 \mathrm{mrad}$. Experiments were carried out at $\sim 65 \mathrm{~K}$, in a vacuum of $10^{-6}$ mbar. The samples were oriented with the $\left[\begin{array}{lll}-1 & 1 & -2\end{array}\right]$ crystal direction parallel to the main axis of the spectrometer which selects the average integration direction. The $p_{x}$ and $p_{y}$ directions of the detector plane were parallel to the [111] and $[-110]$ crystal axes, respectively.

\section{RESULTS AND DISCUSSION}

Positron annihilation lifetime spectroscopy (PALS) spectra taken on both electron-irradiated samples revealed a single component with a lifetime of $245 \pm 3$ ps. This is in good agreement with the work of Mäkinen et al. ${ }^{10}$ who found a $248 \pm 2$ ps single component in similar electronirradiated material. These authors gave convincing evidence that this lifetime was coming from saturation trapping into the $\mathrm{V}_{\mathrm{Si}}: \mathrm{P}$ defect and here we refer the reader to their original work for the arguments behind this conclusion.

The 2D-ACAR spectra for the as-grown and the electron irradiated samples are shown in Figs. 2(a) and 2(b), respectively. The change in overall momentum pattern is noticeable, there being less distinct features in the pattern after irradiation. The pattern after irradiation is more "circular" and the distribution more "peaked." This peaking is as expected for positrons annihilating more with low momentum valence electrons.

Figures 3(a) and 3(b) show the $B^{2 \gamma}$ functions (the 2D-FT of the 2D-ACAR spectra shown in Fig. 2) for $\mathrm{Si}$ and the E-center along the [110] and [111] directions. As we have seen, for delocalized electrons in a regular lattice, $B^{2 \gamma}(\mathbf{r}$ $\left.=\mathbf{m}_{\mathbf{L}}\right)=0 .{ }^{11}$ The positron-electron correlations present in 

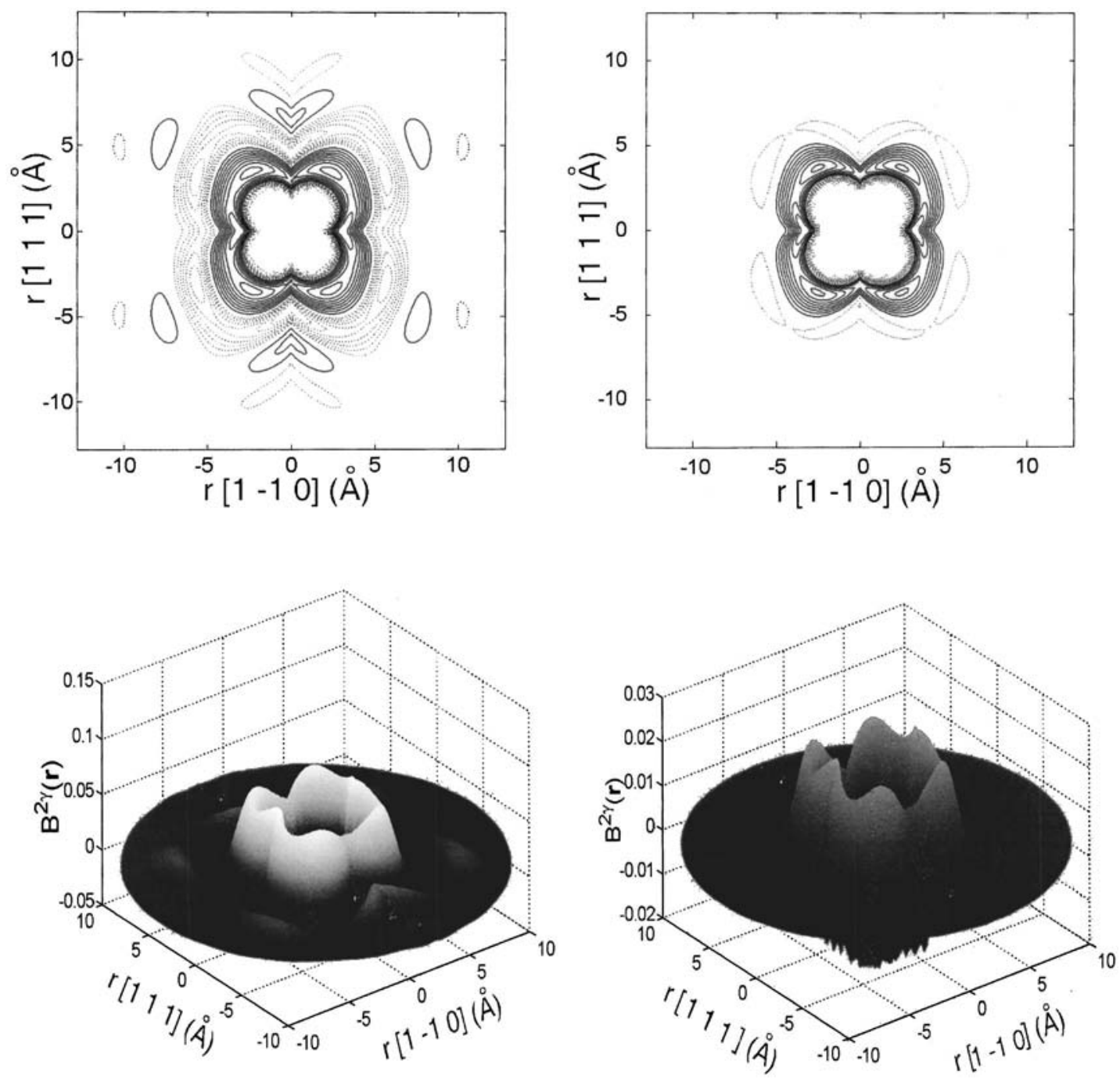

FIG. 3. Autocorrelation functions $B^{2 \gamma}(x, y)$ on the (-211) plane in (a) heavily P-doped Si and for (b) positrons trapped in the E-center. (Note that the vertical scale on the 3D relief plot for the defected sample has been expanded.)

ACAR studies are found to shift the zero-crossing positions of the $B^{2 \gamma}$ functions outward fractionally by a few percent. ${ }^{4,12}$ This effect can be seen clearly in the [110] direction (Fig. 4) in which the $B^{2 \gamma}(\mathbf{r})$ "ripples" are greatest in intensity. The zero-crossing positions are slightly outward displaced. (The first zero crossing, which occurs at $0.5 a_{110}$ $=3.84 \AA, 5,13$ is $\sim 2 \%$ outward displaced.) This is in good accord with the previous observations of the positronelectron correlation effect. ${ }^{4,12}$ In the [111] direction the Bravais vector $a_{111}$ is too far out and in the spectral noise making any definite statement concerning the zero-crossing position in the [111] direction impossible. However, the fact that the "ripples" are less in the [111] direction is consistent with theory. ${ }^{5}$

It may be seen from Fig. 4 that in both [110] and [111] directions that the $B^{2 \gamma}(\mathbf{r})$ zero-crossings for the E-center are displaced outwards relative to those for the bulk state. The displacement is quite small in the [110] direction $(\sim 2 \%)$ and appears larger in the [111] direction $(\sim 7 \%)$, although the effect of noise on the latter zero-crossing could be significant. Another observation, which is difficult to state with too much certainty owing to the limited accuracy of the data, is that it appears that in the [1-10] direction there is only a significant outward shifting at the first zero crossing $\left(0.5 a_{110}\right)$ while for the second and larger zero-crossings the outward shift is either much smaller or zero. The effect of outward shifting of zero-crossings for the positron trapped in a defect has been found theoretically. ${ }^{2}$ Although the reason for the shifting is not completely understood at present, the suggestion is made below that it is associated with those annihilations coming from the lower momentum localized orbitals of the defect.

With the anticipation that the $B^{2 \gamma}(\mathbf{r})$ for the irradiated sample contains structural information on the E-center, $B^{2 \gamma}(\mathbf{r})$ is obtained in the plane defined by the [1-10] and [111] directions by direct 2D Fourier transform of the ACAR data. ${ }^{14}$ The resulting polar $B^{2 \gamma}(x, y)$ plots for "as-grown" and defected samples are shown in Figs. 3(a) and 3(b), respectively. The striking feature of these plots is that although the magnitude of the "ripples" are much lower for the defected sample (i.e., the $\mathrm{V}_{\mathrm{Si}}: \mathrm{P}$ defect), the shape of the plot remains similar with peaks occurring in approximately the same radial locations. Closer inspection, however, reveals two important details from the $B^{2 \gamma}$ plot of the $\mathrm{V}_{\mathrm{Si}}: \mathrm{P}$ defect. 

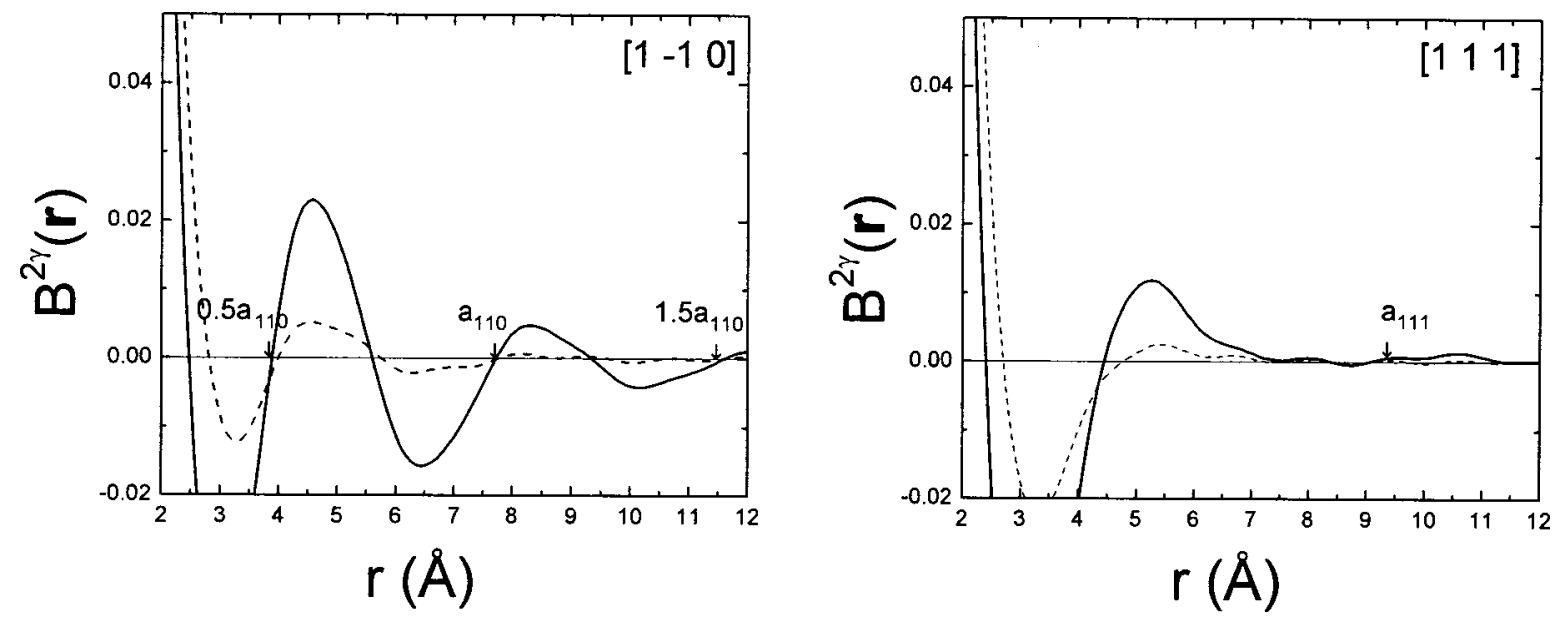

FIG. 4. Autocorrelation function $B^{2 \gamma}(\mathbf{r})$ as measured for (a) the [1-10] direction and (b) the [111] direction. Solid line for as-grown P-doped Si and dashed line for positrons trapped at the E-center.

First the secondary peaks (in the 5-10 $\AA$ range) are much diminished-so much so that they are not visible on the contour plot. Second the primary peak positions are all slightly displaced outwards-a fact that concurs with the outward shifting of the zero-crossings on the 1D plots of Fig. 4.

The strong attenuation of the secondary peaks finds natural explanation on the basis of the localized positron wave function in the defect; i.e., with the positron localized in space, it is clear that neither it, nor the derived positronelectron wave product can autocorrelate significantly at large distances. Kobayasi has shown that the electron-positron autocorrelation function $B^{2 \gamma}(\mathbf{r})$ is related to the electron autocorrelation function $B(\mathbf{r})$ as obtained in Compton profile measurement via the "averaged positron autocorrelation function" $\bar{P}(\mathbf{r})$ in a simple product form ${ }^{15}$

$$
B^{2 \gamma}(\mathbf{r})=\bar{P}(\mathbf{r}) B(\mathbf{r}),
$$

where $\bar{P}(\mathbf{r})$ is given by

$$
\bar{P}(\mathbf{r})=\int F(\mathbf{s}, \mathbf{r}) \psi_{+\mathrm{loc}}^{*}(\mathbf{s}) \psi_{+\mathrm{loc}}(\mathbf{r}+\mathbf{s}) d \mathbf{s}
$$

and

$$
F(\mathbf{s}, \mathbf{r})=\frac{\Sigma_{j} \psi_{j}^{*}(\mathbf{s}) \psi_{j}(\mathbf{s}+\mathbf{r})}{\sum_{j} \int \psi_{j}(\mathbf{s}) \psi_{j}(\mathbf{s}+\mathbf{r}) d \mathbf{s}},
$$

being the normalized electron weight function. ${ }^{15}$ The present work does not attempt the difficult task of calculating $F(\mathbf{s}, \mathbf{r})$ but points out that if this function were close to unity over most of the integration space of the integral in Eq. (2) then a tractable analytical form results. Specifically if one takes $F(\mathbf{s}, \mathbf{r})=1$ then $B_{\text {def }}^{2 \gamma}(\mathbf{r})$ for the defect state can be approximated by assuming some reasonable form for the localized positron wave function $\psi_{+\mathrm{loc}}(\mathbf{r})$. In the first approximation the positron can be considered as trapped in a square well, in which case $\psi_{+ \text {loc }}(\mathbf{r})$ will decay radially as $\exp (-\alpha|\mathbf{r}|) /(\alpha|\mathbf{r}|)$ outside the defect, $\alpha$ being the decay parameter. The autocorrelate $\bar{P}(\mathbf{r})$ of this form of $\psi_{+ \text {loc }}(\mathbf{r})$ is, after performing the integration in spherical coordinates, simply

$$
\bar{P}(\mathbf{r})=e^{-\alpha|\mathbf{r}|} .
$$

In Fig. 5 we plot the ratio $B_{\text {def }}^{2 \gamma}(\mathbf{r}) / B^{2 \gamma}(\mathbf{r})$ for the [1-10] direction. It is known that $B^{2 \gamma}(\mathbf{r})$ is close in form to that of the electron wave-function autocorrelate $B(\mathbf{r})$, and so the plot of Fig. 5 is expected to give $\bar{P}(\mathbf{r})$. Apart from those portions of the graph which asymptote to infinity as a result of zero division (accentuated by the outward zero-crossing shifts), a close to exponential form for $\bar{P}(\mathbf{r})$ is found, with $\alpha$ $=0.29 \AA^{-1}$. This observation is supportive of the above theory and suggests tentatively taking the further step of estimating the positron binding energy to the defect from the value of $\alpha$ which for a particle bound to a square well with energy $E_{B}$ is given as

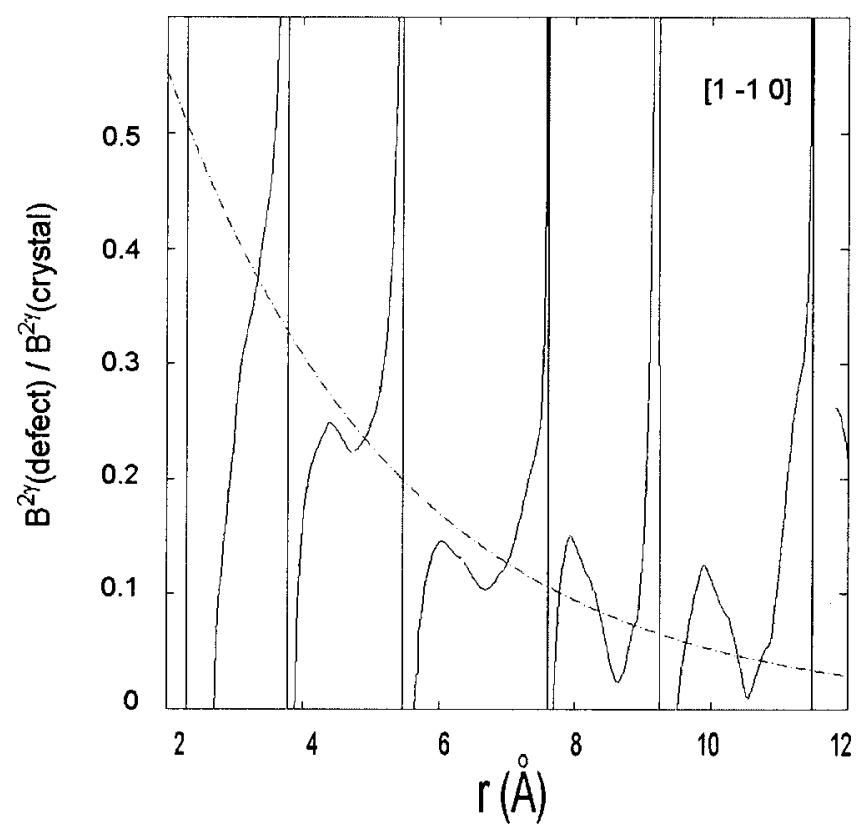

FIG. 5. Ratio of $B^{2 \gamma}(\mathbf{r})$ functions $B_{\text {def }}^{2 \gamma}(\mathbf{r}) / B^{2 \gamma}(\mathbf{r})$ in the [1-10] direction. The dashed line gives the positron autocorrelate $\bar{P}(r)$ as described by Eq. (9). 


$$
\alpha=\frac{1}{\hbar c} \sqrt{2 m c^{2} E_{B}} .
$$

Taking $m^{*}$, the positron effective mass, as the bare electron mass ${ }^{16}$ this equation gives a positron binding energy $E_{B}$ $=0.22 \mathrm{eV}$. This value, in spite of differing from the calculated $E_{B}$ of $0.61 \mathrm{eV}$ for the negatively charged Si vacancy, is nevertheless of the correct order of magnitude. ${ }^{17}$

Before concluding we make some general observations relating to the original purpose behind the present study as outlined in the Introduction, namely that of trying to extract the component of $B_{\text {def }}^{2 \gamma}(\mathbf{r})$ originating just from localized defect bonds. The above approximate separation of $B_{\text {def }}^{2 \gamma}(\mathbf{r})$ into electron and positron autocorrelates appears to provide some support for such a venture, since it suggests a means of subtracting that component of $B_{\text {def }}^{2 \gamma}(\mathbf{r})$ originating from delocalized valence electrons. We suggest writing Eq. (2) for a trapped positron in the form

$$
\begin{aligned}
B_{\mathrm{def}}^{2 \gamma}(\mathbf{r})= & \sum_{\mathrm{val}} \int \psi_{+\mathrm{loc}, j}^{*}(\mathbf{r}+\mathbf{s}) \psi_{+\mathrm{loc}, j}(\mathbf{s}) d \mathbf{s} \\
& -\sum_{j=1}^{n_{\mathrm{atom}}} \int \psi_{+\mathrm{loc}, j}^{*}(\mathbf{r}, \mathbf{s}) \psi_{+\mathrm{loc}, j}(\mathbf{s}) d \mathbf{s} \\
& +\sum_{n_{\mathrm{loc}}} \psi_{+\mathrm{loc}, j \mathrm{loc}}^{*}(\mathbf{r}+\mathbf{s}) \psi_{+\mathrm{loc}, j \mathrm{loc}}(\mathbf{s}) d \mathbf{s}
\end{aligned}
$$

where the first two terms come from the delocalized valence electrons after having removed the $n_{\text {atom }}$ valence electrons associated with the missing atom of the defect. The final term replaces the $n_{\text {loc }}$ localized dangling bond electrons associated with the defect. Assuming that the small number $n_{\text {loc }}$ of missing states from the total complement of valence band states is not going to effect the term significantly and employing the separability of the first term into positron and electron autocorrelates Eq. (12) may be written as

$$
B_{\mathrm{def}}^{2 \gamma}(\mathbf{r}) \approx \bar{P}(r) B(\mathbf{r})-B_{\mathrm{atom}}^{2 \gamma}(\mathbf{r})+B_{\mathrm{loc}}^{2 \gamma}(\mathbf{r})
$$

Equation (13) raises the question as to whether or not $B_{\operatorname{loc}}^{2 \gamma}(\mathbf{r})$ could be extracted from the observed $B_{\text {def }}^{2 \gamma}(\mathbf{r})$ signal. The presence of the term $B_{\text {atom }}^{2 \gamma}(\mathbf{r})$ certainly complicates the extraction. However, because both $B_{\text {loc }}^{2 \gamma}(\mathbf{r})$ and $B_{\text {atom }}^{2 \gamma}(\mathbf{r})$ involve sums over the same covalent orbitals it may be possible to combine them. This matter demands further theoretical investigation, but it is likely here that Babinet's principal in optics can be applied, which would predict the same diffraction pattern [hence the same $B_{\text {loc }}^{2 \gamma}(\mathbf{r})$ ] for the absence of electron states as for their presence. However, in support of this proposition it is noted that it would explain why the zero passages of $B_{\text {def }}^{2 \gamma}(\mathbf{r})$ move outwards compared to those of $B(\mathbf{r}) . B_{\text {atom }}^{2 \gamma}(\mathbf{r})$ is expected to exceed $B_{\text {loc }}^{2 \gamma}(\mathbf{r})$ in amplitude since more electron states are associated with the atom than the dangling bonds and thus the combination of the last two terms in Eq. (13) would be expected to contribute a total negative component in the region of covalent bonding [i.e., around the first minimum of $\left.B_{\mathrm{def}}^{2 \gamma}(\mathbf{r})\right]$ - such a lowering of the value of $B_{\mathrm{def}}^{2 \gamma}(\mathbf{r})$ would move zero-crossings outwards in the manner we observe.
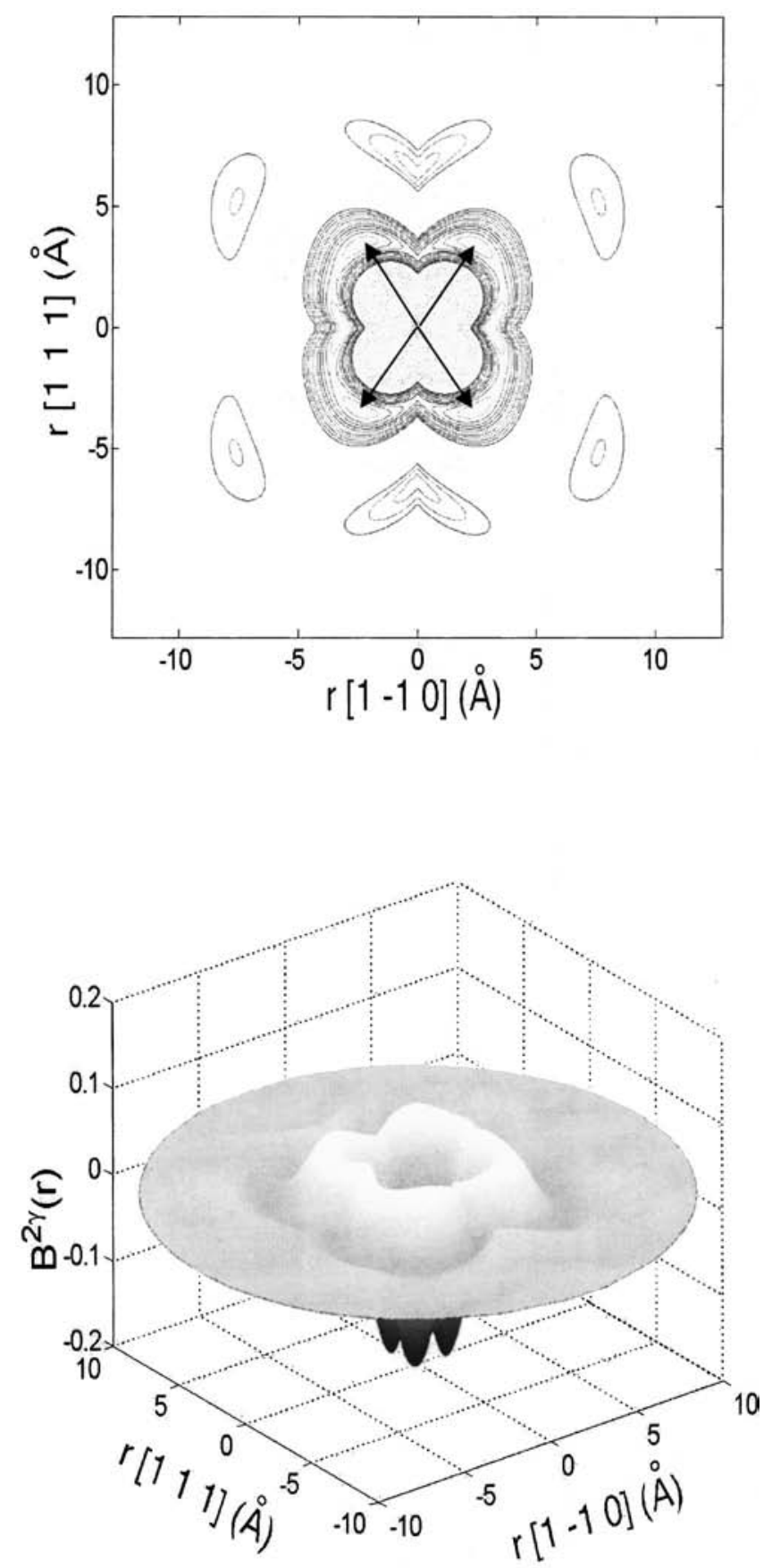

FIG. 6. Approximate $B^{2 \gamma}(x, y)$ autocorrelation function originating from localized electron orbitals at the E-center as obtained using the formulation $\bar{P}(r) B(\mathbf{r})-B_{\text {def }}^{2 \gamma}(\mathbf{r})$. The arrows indicate the expected "A"- "B" autocorrelation peaks due to the nearest neighbor atomic positions in the defect. This type of plot may represent the best real-space inversion of McMullen and Bishop's defect diffraction pattern (Ref. 1).

In order to check the validity of the above arguments we perform the subtraction $\bar{P}(\mathbf{r}) B(\mathbf{r})-B_{\text {def }}^{2 \gamma}(\mathbf{r})$ in the plane defined by the [1-10] and [111] directions by taking $\bar{P}(\mathbf{r})$ in the simple isotropic form given by Eq. (12). The result is shown in Fig. 6. While the complete form of this resultant function is difficult to completely understand, we do draw attention to the positive peaks in the (1-10) and (110) directions. These can be interpreted as the autocorrelates of the closest atomic orbitals "A" and "B" to the missing Si atom (see Fig. 7). 


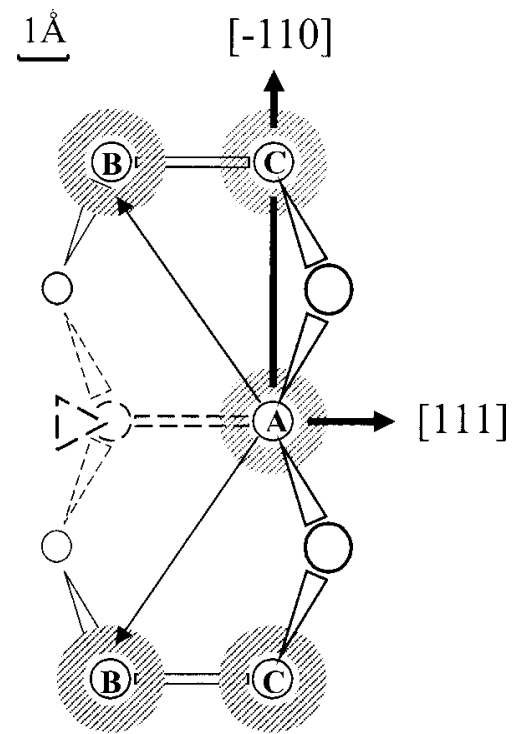

FIG. 7. Diagram showing nonrelaxed positrons of neighboring atoms on the (-211) plane. Atoms A and B are those closest to the vacancy. The dotted lines show the missing atom and bonds associated with the E-center. Atoms $\mathrm{A}, \mathrm{B}$, and $\mathrm{C}$ are those nearest neighbors and the shaded region around them is meant to indicate the region of positron-electron overlap. The vectors shown at $35^{\circ}$ correspond to the positions of maximum autocorrelation as seen in the same directions on the $B^{2 \gamma}(x, y)$ mapping of Fig. 6 .

Not only are the observed peaks in the $\bar{P}(r) \mathbf{B}(\mathbf{r})-B_{\text {def }}^{2 \gamma}(\mathbf{r})$ mapping in the same approximate positions $\left(35^{\circ}\right.$ to the [-110] direction) but the distance $\sim 4.1 \AA$ is close to the expected distance of autocorrelation. The next atomic autocorrelations one might expect would be those of "B" to "C" and " $A$ " to " $C$." The peaking that occurs at $3 \AA$ along the [111] direction may well correspond to the " $\mathrm{B}$ " to " $\mathrm{C}$ " autocorrelation (which would be expected to be close to the $\mathrm{Si}-\mathrm{Si}$ bond length of $2.35 \AA$ ), while the autocorrate " $\mathrm{A}$ "" $C$ " in the $[-110]$ direction, being the weaker of the three, may be obscured by the strong "A"- " $\mathrm{B}$ " signal. Finally it is pointed out that the absence of a dominant central peak in the mapping of Fig. 6, which would be expected for any autocorrelation function, may be due to the oversimplified form of $\bar{P}(\mathbf{r})$ we have used or a breakdown in the separability condition. Theoretical studies on expected positron-electron autocorrelates will probably be the surest route to confirming the above associations and on determining the best procedures for subtracting the autocorrelation due to electron Bloch state annihilations.

\section{CONCLUSIONS}

FT-ACAR positron-electron autocorrelation studies have been carried out on both bulk crystal Si crystal (heavily $n$-type doped) and on the $\mathrm{V}_{\mathrm{Si}}: \mathrm{P}$ defect center. It becomes clear that while much of the defect's autocorrelation function is similar to the bulk, because it is largely governed by annihilations with delocalized valence electrons, there is still some component in the autocorrelation function of the defect coming from localized electron orbitals. The present work has found support for the recent suggestion that the autocorrelation functions of trapped positron and the delocalized electrons in a defect may be separable. This concept lends some understanding to the observed increased damping of the $B^{2 \gamma}(\mathbf{r})$ function observed in the case of positrons trapped at the E-center defect. Employing this separability approximation we have obtained a reasonable value for the binding energy of the positron to the E-center that supports this general picture. Using the same approximation it has been possible to approximately isolate that part of the measured $B^{2 \gamma}(\mathbf{r})$ function that originates from the localized defect orbitals. Some of the features of this functional mapping find natural interpretation based on the approximate known positions for atoms in the E-center. There is thus some optimism that this type of mapping could form a future basis of obtaining some elementary "image" of localized orbitals associated with vacancy type defects.

\section{ACKNOWLEDGMENTS}

The work described in this article is partially supported by the grants from the Research Grant Council of the Hong Kong Special Administrative Region, China (under Project Nos. HKU7103/02P, HKU7104/98P, HKU7091/00P, and HKU1/00C) and the Hung Hing Ying Physical Science research fund. M. Gong also acknowledges support from the grant of National Nature Science of China (No. 60076010) and the grant of the National Key Lab of Silicon Materials of China. The authors would like to thank P. Y. Kwan for preparing the graphs.

${ }^{1}$ T. McMullen and M. F. Bishop, Phys. Rev. B 55, 4046 (1997).

${ }^{2}$ C. D. Beling, W. LiMing, Y. Y. Shan, S. H. Cheung, S. Fung, B. K. Panda, and A. P. Seitsonen, J. Phys.: Condens. Matter 10, 10475 (1998).

${ }^{3}$ S. Berko, in Positron Solid State Physics, Proceedings of the International School Phys. "Enrico Fermi" Course 83 (IOS, Amsterdam, 1983), p. 76.

${ }^{4}$ S. Berko, W. S. Farmer, and F. Sinclair, in Positron Annihilation, edited by P. G. Coleman, S. C. Sharma, and L. M. Diana (North Holland, Amsterdam, 1982), p. 319.

${ }^{5}$ P. Pattison and J. R. Schneider, Solid State Commun. 28, 581 (1978).

${ }^{6}$ B. Kramer, P. Krusius, W. Schröder, and W. Schülke, Phys. Rev. Lett. 38, 1227 (1977).

${ }^{7}$ R. N. West, in Positron Spectroscopy of Solids, Proceedings of the International School Phys. "Enrico Fermi" Course CXXV (IOS, Amsterdam, 1995), p. 75.

${ }^{8}$ W. Schülke, Phys. Status Solidi B 82, 229 (1977).

${ }^{9}$ R. Benesch, S. R. Singh, and V. H. Smith, Jr., Chem. Phys. Lett. 10, 151 (1971).

${ }^{10}$ J. Mäkinen, C. Corbel, P. Hautojärvi, P. Moser, and F. Pierre, Phys. Rev. B 39, 10162 (1989).

${ }^{11}$ M. Biasini, G. Ferro, M. A. Monge, G. Di Francia, and V. La Ferrara, J. Phys.: Condens. Matter 12, 5961 (2000).

${ }^{12}$ B. K. Panda and D. P. Mahapatra, J. Phys.: Condens. Matter 5, 3475 (1993).

${ }^{13}$ A. Seth and D. E. Ellis, J. Phys. C 10, 181 (1977).

${ }^{14}$ S. Berko, in Positron Solid State Physics, Proceedings of the International School Phys. "Enrico Fermi" Course 83 (IOS, Amsterdam, 1983), p. 83.

${ }^{15} \mathrm{~T}$. Kobayasi, in Electron, Spin and Momentum Densities and Chemical Reactivity, edited by P. G. Mezey and B. E. Robertson (Kluwer Dordrecht, 2000), p. 169; Mater. Sci. Forum 363-365, 689 (2001).

${ }^{16}$ E. Soininen, J. Makinen, D. Beyer, and P. Hautojarvi, Phys. Rev. B 46, 13104 (1992).

${ }^{17}$ S. Mäkinen and M. J. Puska, Phys. Rev. B 40, 12523 (1989). 